\title{
Identification of Novel Variants in MEN1: A Study Conducted with Four Multiple Endocrine Neoplasia Type 1 Patients
}

Authors

Wen-mu Hu, Qin Zhang, Li-hua Huang, Zhao-hui Mo, Xiao-dan Long, You-bo Yang, Wen-jun Yang, Jun Liu, Ping Jin

\begin{abstract}
Affiliation
Department of Endocrinology, The Third Xiangya Hospital, Central South University, Changsha, Hunan, China
\end{abstract}

Key words

multiple endocrine neoplasia type 1, adenomas, variant

received $\quad 05.02 .2020$

accepted after revision $\quad 18.03 .2020$

published online $\quad 16.04 .2020$

Bibliography

Horm Metab Res 2020; 52: 788-795

DOI 10.1055/a-1147-1375

ISSN 0018-5043

(c) 2020. Thieme. All rights reserved.

Georg Thieme Verlag KG, Rüdigerstraße 14,

70469 Stuttgart, Germany

Correspondence

Ping Jin PhD, MD

Department of Endocrinology, The Third Xiangya Hospital,

Central South University

410007 Changsha

Hunan

China

Tel.: + 86731886501865 , Fax: + 8673188618006

jping7676@hotmail.com

Supplementary material is available under

https://doi.org/10.1055/a-1147-1375.

\section{ABSTRACT}

Multiple endocrine neoplasia type 1 (MEN1) is an autosomal dominant inherited endocrine tumor syndrome caused by inactivating variants of the MEN1 gene. The aim of this study is to explore the clinical and genetic characteristics of four MEN1 patients. We isolated genomic deoxyribonucleic acid from lymphocytes, parathyroid, and thymic tumoral tissue specimens from the MEN1 patients. All exons of the MEN1 and CDNK1B genes and adjacent exon-intron sequences were amplified by polymerase chain reaction and subsequently sequenced. Further, the splice alterations were studied by sequencing the amplified RT-PCR products for MEN1 CDNA. We identified four heterozygous MEN1 germline variants: c.564delC, c.1268G > A, IVS5 + 5delG, and c.1546_1547insC. Both c.564delC and IVS5 + 5 delG were novel variants. The impact of the MEN1 splice variant, IVS5 + 5delG, was evaluated using bioinformatics and in vitro analyses. The analyses indicated that this variant resulted in skipping of the neighboring exon and was disease-causing. Two novel somatic variants, c.249_252delGTCT and c.313_314insC, were found. Additionally, loss of heterozygosity $(\mathrm{LOH})$ for the MEN1 locus (IVS5 + 5delG and c.564delC) was found in tumor tissue samples from the MEN1 patients, consistent with Knudson's two-hit mechanism. We identified four MEN1 germline variants and two novel somatic variants. Early recognition of the phenotype coupled with variant screening of the MEN1 gene is the key to diagnosing and treating MEN1 effectively at an early stage.

\section{Introduction}

Multiple endocrine neoplasia type 1 (MEN1; OMIM 131100) is a rare hereditary autosomal dominant disorder with an incidence of $1 / 30000-50000$ [1]. It is characterized by the occurrence of parathyroid adenomas (95\%), pituitary adenomas (30-40\%), and gastrointestinal pancreatic neuroendocrine neoplasia (40-70\%) [2]. MEN1 is caused by an inactivating variant of the MEN1 (OMIM 613733) gene, which is located on chromosome 11 q13 and encodes a 610-amino acid protein called menin [3]. Menin is a scaffold protein that interacts with other intracellular molecules including JunD, NFkB, and Smad3, which are involved in transcriptional regulation, genome stability, cell division, and proliferation [3].
More than 1200 germline variants in the MEN1 gene have been identified including frame-shift variants (42\%), missense/nonsense variants (39.5\%), splicing variants (10.5\%), large deletions (2.5\%), and in-frame deletions or insertions (5.5\%) [4]. There is no specific hotspot but MEN1 variants are distributed across the entire gene. MEN1 is a tumor-suppressor gene and MEN1 patients have a heterozygous germline variant in the gene that acts as the first 'hit.' Subsequently, somatic variant leads to the loss of heterozygosity $(\mathrm{LOH})$ in the tumor suppressor role for menin, which is consistent with the Knudson's two-hit hypothesis [5].

Approximately $10-25 \%$ of MEN1 patients may lack variants in the MEN1 gene [4]. Recently, a germline variant in the cyclin-de- 
pendent kinase inhibitor (CDNK)1B/p27 tumor suppressor gene was identified in a family that presented with pituitary tumors, parathyroid adenoma, renal angioleiomyoma, and testicular cancer [6]. The MEN1-like syndrome attributed to these variants was named MEN4 $[7,8]$. However, only 19 cases of MEN4 including 16 different variants have been reported. In addition, $2 \%$ of the patients may have variants in the CDKN family, such as CDKN1A, CDKN2B, and $C D K N 2 C$, which overlap with the clinical manifestations of MEN1 $[4,8]$.

In our study, we explored the clinical and genetic characteristics of four MEN1 patients. We identified four heterozygous MEN1 germline variants (c.564delC, c.1268G > A, IVS5 + 5delG, and c.1546_1547insC), two novel somatic variants (c.249_252delGTCT and c.313_314insC) and LOH for IVS5 + 5delG and c.564delC in tumor tissues from the MEN1 patients.

\section{Patients and Methods}

\section{Ethical statement}

We conducted this study with four MEN1 patients. We collected the peripheral blood as well as surgically resected parathyroid and thymic tumor tissue from the patients. Further, peripheral blood samples from the pedigree and 100 unrelated healthy controls were also collected. All procedures were in accordance with the World Medical Association's Declaration of Helsinki. We obtained approval for this study from the Institutional Ethics Committee of the Third Xiangya Hospital. We also obtained written informed consent from all the subjects enrolled in this study.

\section{Patient's data}

\section{Patient \#1}

Patient \#1 was a 32-year-old woman suffering from amenorrhea for 5 years. She had a history of nephrolithotomy. Laboratory tests revealed hypercalcemia, hypophosphatemia, and high parathyroid hormone (PTH) levels. Further, her parathyroid ultrasonography indicated bilateral parathyroid adenoma and urinary ultrasonography suggested multiple bilateral kidney stones. Magnetic resonance imaging (MRI) revealed a microadenoma, which was presumed to be a prolactinoma due to hyperprolactinemia. Abdominal computed tomography $(\mathrm{CT})$ revealed a left adrenal nodule with a size of $0.7 \times 0.4 \mathrm{~cm}$. Her mother had been diagnosed with MEN1, including parathyroid adenoma, pituitary prolactinoma, and insulinoma. A MEN1 diagnosis was made because she had MEN1-associated tumors and a first-degree relative with MEN1 ( $\triangleright$ Table 1). She underwent subtotal parathyroidectomy (3.5 glands). The pathological findings were consistent with parathyroid adenoma. Calcium and Vitamin D supplements were given and the prolactinoma was treated with $0.5 \mathrm{mg}$ cabergoline twice a week as bromocriptine showed poor efficacy.

\section{Patient \#2}

Patient \#2 was a 25-year-old woman suffering from parathyroid adenoma for 6 months. She reported having a pituitary tumor resection at the age of 15 and the pathological results indicated a prolactinoma. Her laboratory tests revealed hypercalcemia, high PTH levels, and nephrolithiasis. She underwent minimally invasive par- athyroidectomy. The pathological findings were consistent with parathyroid adenoma. However, she still had persistent hypercalcemia and high PTH levels after the operation. She underwent subtotal parathyroidectomy 4 months later and her plasma PTH and calcium levels were normal after the surgery, indicating the surgery was successful. No consanguinity was found in her parents.

\section{Patient \#3}

Patient \#3 was a 38-year-old man who came to our hospital with episodes of hypoglycemia lasting for 16 years. He was diagnosed with insulinoma in 2002 and underwent his first operation for insulinoma. Subsequently, he experienced a recurrence of hypoglycemia in 2010 and hence underwent laparoscopic distal pancreatectomy and splenectomy. However, he developed symptoms of hypoglycemia after 2 years. His laboratory tests confirmed persistent hypoglycemia with hyperinsulinism. A $9 \mathrm{~mm}$ lesion was found at the head of the pancreas with the help of pancreatic CT. Hypercalcemia and high PTH levels were also found. Parathyroid ultrasonography revealed bilateral parathyroid adenomas. Pulmonary CT revealed thymoma with a size of $9.1 \times 7.5 \times 8.9 \mathrm{~cm}$. Adrenal CT showed bilateral adrenal nodules. A MEN1 diagnosis was made because he had three MEN1-associated tumors ( $>$ Table 1 ). He underwent surgical resection of insulinoma once again. However, the patient continued to suffer from hypoglycemia and refused a total pancreatectomy, which was recommended by the physicians. Thymoma resection surgery was performed in 2018. The pathological examination suggested a thymic carcinoid with a pathology stage of G2 for the thymoma (karyokinesis < 10/10HPF, ki-67 20\%). He also underwent subtotal parathyroidectomy (3.5 glands) in 2019. The pathological findings were consistent with parathyroid adenoma. No consanguinity was found in his parents.

\section{Patient \#4}

Patient \#4, a 52-year-old woman, visited our hospital because of recurrent kidney stones since 2012 . Her laboratory tests revealed anemia, hypercalcemia, high serum creatinine $(\mathrm{Cr})$ and high PTH levels. Parathyroid ultrasonography showed bilateral parathyroid adenomas. She was first diagnosed with chronic renal insufficiency and tertiary hyperparathyroidism. She underwent minimal parathyroidectomy and the pathological examination showed adenomatous hyperplasia of the parathyroid glands. However, she continued to have hypercalcemia, high PTH, and high Cr levels. Later, subtotal parathyroidectomy was performed in March 2013. In 2019, the patient visited our hospital as she was suffering from gastrointestinal bleeding. Her gastroscopy report revealed multiple ulcers in the gastroduodenum. The pathological examination suggested neuroendocrine neoplasia with $\mathrm{CgA}(++)$, syn (+), and ki-67 $(<2 \%)$. She had a high gastrin-17 level but a normal insulin release index. Abdominal CT revealed left adrenal adenoma and multiple pancreatic tumors, which led to a high suspicion of gastrinoma. MRI revealed the presence of a $2.0 \times 2.5 \times 2.5 \mathrm{~cm}$ large pituitary adenoma, which was presumed to be a prolactinoma due to hyperprolactinemia. A MEN1 diagnosis was established because she had three MEN1-associated tumors ( $>$ Table 1 ). An operation was not performed as she was prone to high risk due to surgery. Instead, she received a proton pump inhibitor, somatostatin, bromocriptine, and hemodialysis treatment. Her father died at a young age 


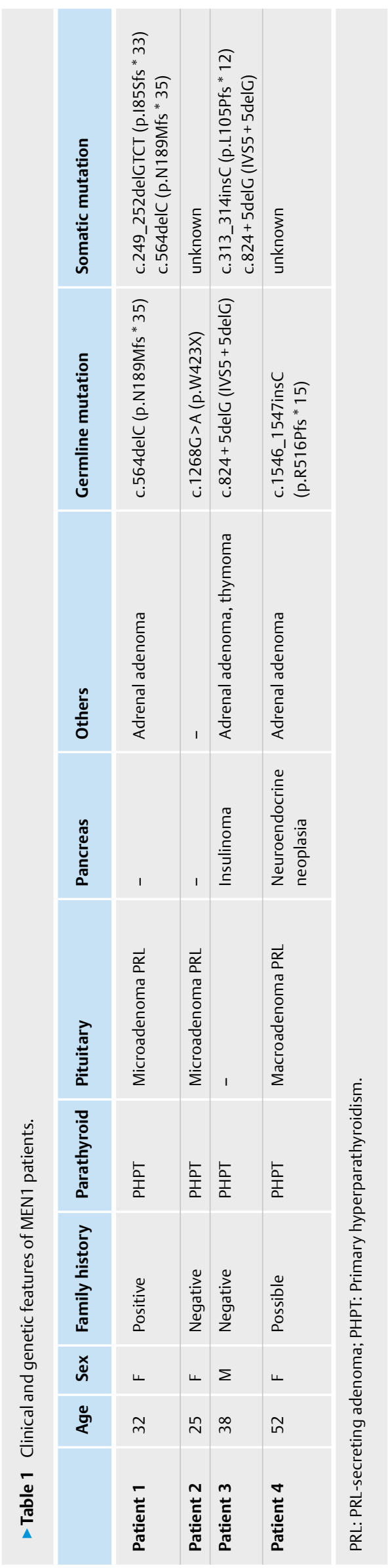

due to gastrointestinal bleeding. Her sister suffered from kidney stones and had pituitary tumor resection. However, her sister declined any further examination; it is unknown whether she had MEN1-related diseases.

\section{MEN1and CDKN1B gene variant analysis}

Genomic DNA was extracted from peripheral blood leukocytes as well as the parathyroid and thymic tumoral tissues using standard phenol-chloroform procedures. All exons and adjacent exon-intron sequences of the MEN1 gene (NM_130799.2) and the CDKN1B gene (NM_004064.4) were amplified by polymerase chain reaction (PCR) using the primers listed in ( $\triangleright$ Table $1 S$ ). Direct sequencing of PCR products on an $A B I 3730 x$ lautomated sequencer helped in identifying the variants (Applied Biosystems, USA). American College of Medical Genetics (ACMG) standards were used to interpret the variants [9]. The variants were categorized as pathogenic, likely pathogenic, variants of uncertain significance (VUS), likely benign, or benign.

\section{Whole exome sequencing}

Because only a splice variant was found in patient \#3 by Sanger sequencing and the splice variant was not located in the first or second positions of the splice acceptor sequence, whole exome sequencing was carried out to exclude other genes associated with MEN1. The isolated DNA was sheared on a Bioruptor UCD-200 (Diagenode) with a size distribution peak around 200 bp. The KAPA Library Preparation Kit (Kapa Biosystems, KR0453) was used to prepare the DNA libraries. Agilent SureSelectXT2 Target Enrichment System's guidelines were followed for the removal of non-hybridized library molecules as well as for the hybridization of pooled libraries to the capture probes. Illumina specifications were followed for sequencing, sample dilution, and flow cell loading. DNA libraries were sequenced on the HiSeq 2500 (Illumina, San Diego, CA, USA) platform as paired-end 200-bp reads. The BWA Aligner was used to filter and align the raw data (hg19) (http://bio-bwa.sourceforge.net/). We estimated the causative variant to be missense, gene-disrupting, and a rare occurrence with less than $1 \%$ frequency in the 1000 Genomes (http://browser.1000genomes.org), Exome Aggregation Consortium (ExAC, http://exac.broadinstitute. org/), and Genome Aggregation Database (gnomAD, http://gnomad.broadinstitute.org/). The potential effects of the variants were then assessed using SIFT (http://sift.jcvi.org), Polyphen-2 (http:// genetics.bwh.harvard.edu/pph2/), Mutation Taster (http://www. mutationtaster.org/), Phylop, phastCons (http://compgen.bscb. cornell.edu/phast/), and Human Splicing Finder (http:// www.umd. be/HSF/) software.

\section{Total RNA preparation and MEN1 cDNA analysis}

To determine the effect of this splice variant on the mRNA level, a high purity Ribonucleic acid (RNA) Isolation Kit (Omega, USA) was used to isolate total RNA from the parathyroid tumor tissues of patient \#3. The isolated RNA was reverse transcribed using the cDNA synthesis kit (ToYoBo, Japan). Primers were chosen that could amplify MEN1 CDNA, 5'-CTTCCATTGACCTGCACACC-3' and 5' - CAGCCAGGTACATGTAGGGG-3', and a 249-bp fragment was amplified. The fragments were sequenced using the $A B I 3730 x$ automated sequencer (Applied Biosystems, USA). 
Immunohistochemistry

Sections of parathyroid tumor were collected from MEN1 patient \#1, patient \#2, and patient \#3. Samples from patients with parathyroid hyperplasia without MEN1 variants were employed as positive controls. Menin expression in the resected tumor specimens was analyzed by immunohistochemistry using the C-terminal antimenin antibody (rabbit no. ab92443, 1:150, Abcam, Cambridge, UK).

\section{Results}

\section{MEN1 germline variant analysis}

Patient \#1 showed a heterozygous insertion variant, c.564delC (p.N189Mfs * 35), in exon 3 of the MEN1 gene ( Fig. 1a). This variant was predicted to generate a truncated protein and a premature termination codon in exon 4. Co-segregated analysis confirmed that this variant was also found in her mother, who had the
MEN1 phenotype. According to the ACMG guidelines, this variant was interpreted as pathogenic.

Patient \#2 showed a heterozygous nonsense variant, c. 1268G > A (p.W423X), in exon 9 of the MEN1 gene ( $\triangleright$ Fig. 1b). This variant was predicted to change the amino acid glutamine at position 423 to a stop codon in exon 9 and has been reported as pathogenic [10]. Co-segregated analysis showed her father also had this variant, but he declined any further examination; it is unknown whether he had MEN1-related diseases. According to the ACMG guidelines, variant c. 1268G > A in the MEN1 gene was interpreted as pathogenic.

Patient \#3 showed a heterozygous variant, c.824 + 5 delG (IVS5 + 5delG), in intron 5 of MEN1 ( $>$ Fig. 1c) with Sanger sequencing. Whole exome sequencing confirmed this variant. Co-segregated analysis showed this variant did not exist in his parents. According to the ACMG guidelines, variant IVS5 + 5delG in the MEN1 gene was interpreted as VUS. Meanwhile, patient \#3 showed a heterozygous polymorphism, c.1621G >A (p.A541T), in exon 10 of the MEN1 gene. His father and sister had homozygous and heterozy-
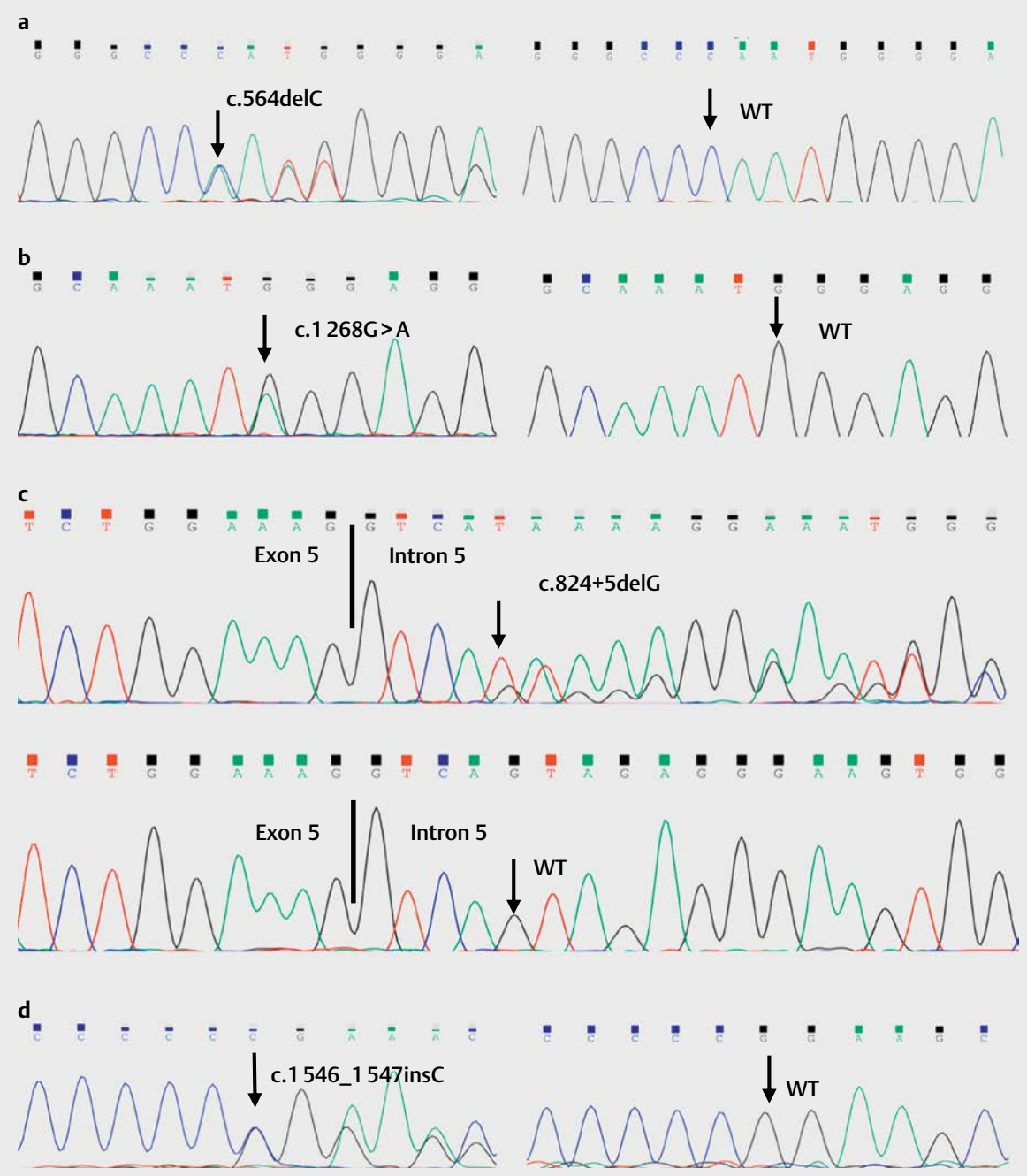

- Fig. 1 Sanger sequencing chromatograms for the MEN1 germline mutations. The arrow indicates the mutation site. a A heterozygous c.564delC mutation was identified in patient \#1. b A heterozygous c.1268G > A mutation was identified in patient \#2. c A heterozygous IVS5 + 5delG mutation was identified in patient \#3. $\mathbf{d}$ A heterozygous c.1546_1547insC mutation was identified in patient \#4. 
gous c. 1621G > A polymorphisms, respectively. No MEN1-related tumors were found in his parents and sister.

Patient \#4 showed a heterozygous variant, c.1546_1547insC (p.R516Pfs * 15), in exon 10 of the MEN1 gene ( $>$ Fig. 1d). This variant might generate a truncated protein. According to the ACMG guidelines, variant c.1546_1547insC was interpreted as pathogenic. The IVS5 + 5 delG and c.564delC variants were not found in 100 ethnically matched controls. Database searches of HGMD (http:// www.hgmd.cf.ac.uk/), dbSNP (http://www.ncbi.nlm.nih.gov/snp), and ClinVar (http://www.ncbi.nlm.nih.gov/clinvar) indicated that they were novel variants.

\section{MEN1 tumor somatic variants analysis}

In one of the parathyroid tumoral tissue samples from patient \#1, we identified a heterozygous c.564delC germline variant and a novel heterozygous somatic c.249_252delGTCT (p.185Sfs * 33) MEN1 variant ( $\mathbf{F i g}$. 2a). The c.249_252delGTCT variant might generate a truncated protein and a premature termination codon in exon 2. LOH of the MEN1 locus C.564delC was detected in another two parathyroid tumoral tissue samples ( $\mathbf{F i g} \mathbf{2} \mathbf{2 b}$ ).

In the thymoma specimen from patient \#3, we identified a heterozygous IVS5 + 5delG germline variant and a novel heterozygous somatic MEN1 variant, c.313_314insC (p.L105Pfs * 12) (- Fig. 2c). The c.313_314insC somatic variant might generate a truncated protein and a premature termination codon in exon 2 . In addition, the LOH of MEN1 IVS5 + 5delG was identified in all parathyroid tumoral tissue samples from patient \#3 ( $\mathbf{F i g}$. 2 d).

\section{Analysis of MEN1 IVS5 + 5delG in silico and in vitro}

The IVS5 + 5delG variants were predicted to be "disease-causing," with Phylop values of 3.654. The variant sites were predicted and conserved with phastCons with a value of 0.99. Additionally, Human Splicing Finder indicated this variant was the most likely to occur.
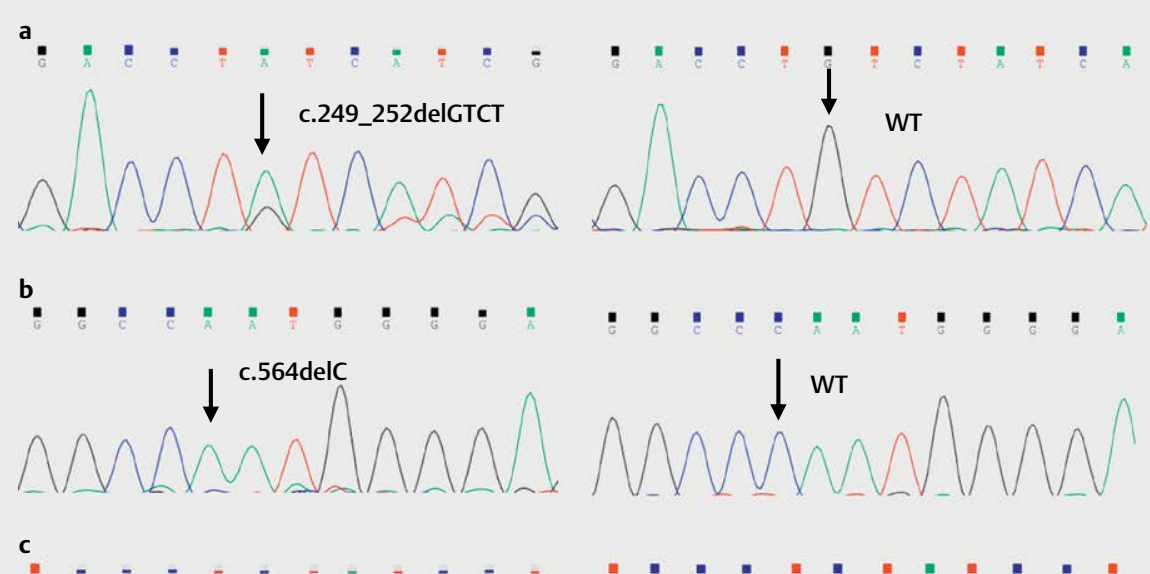

c
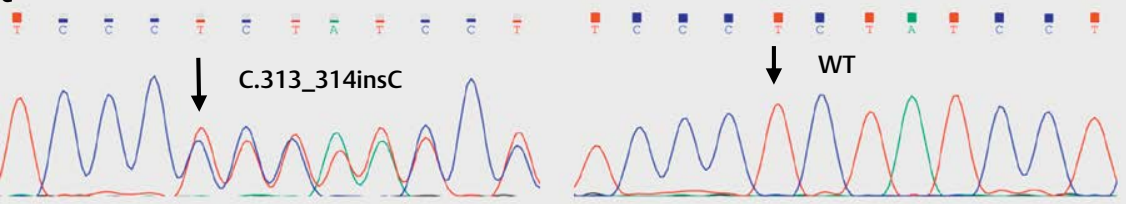

d
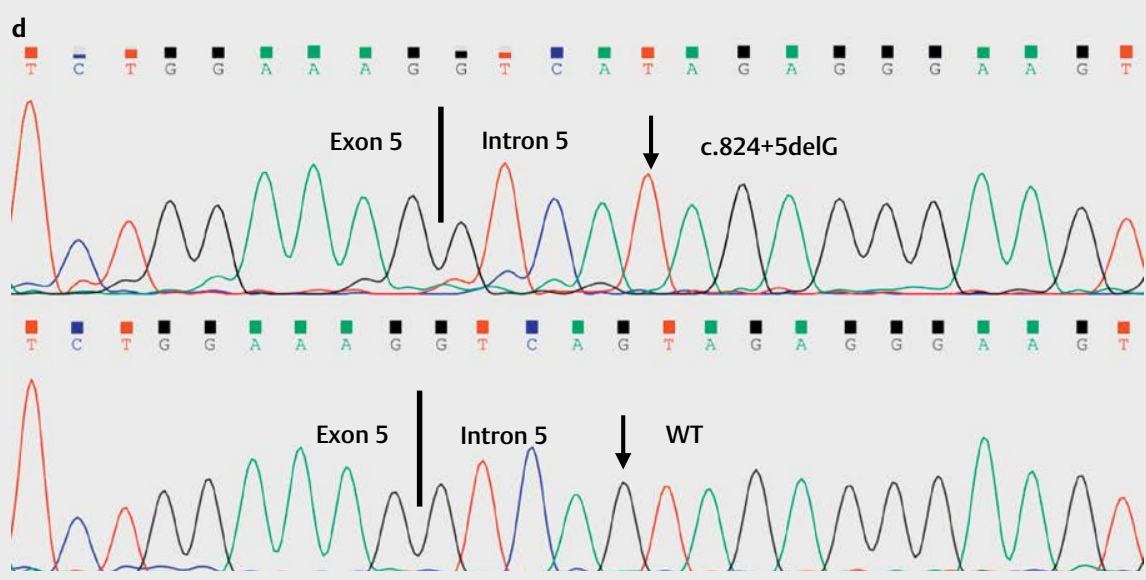

Fig. 2 Sanger sequencing chromatograms of the MEN1 somatic mutations in the surgically resected tissue samples. The arrow indicates the mutation site. a A heterozygous c.249_252delGTCT mutation was identified in one of the parathyroid tissues from patient \#1. b LOH of the MEN1 c.564delC mutations was detected in another two parathyroid tissue samples from patient \#1. c A heterozygous c.313_314insC mutation was detected in the thymoma of patient \#3. D: LOH of MEN1 IVS5 + 5delG mutations was identified in all the parathyroid tissues from patient \#3. 
We performed RT-PCR for MEN1 cDNA to confirm the presence of aberrant RNA transcript splice products. Sequencing of the amplified RT-PCR products showed the IVS5 + 5 delG variant resulted in the skipping of exon 5 ( $\mathbf{F i g . 3 a , ~ b ) , ~ w h i c h ~ r e s u l t e d ~ i n ~ a ~ d o w n - ~}$ stream stop codon with premature termination of translation.

\section{CDKN1B gene variant analysis}

No CDKN1B gene variants were found in any of the patients.

\section{Immunohistochemistry}

Menin nuclear staining in control patients with renal failure is shown in > Fig. 4 a along with reduced nuclear menin expression in patient \#1, patient \#2, and patient \#3 ( $\triangleright$ Fig. 4 b-d).

\section{Discussion}

Menin is a nuclear protein with three nuclear localization signals (NLSs) located in its C-terminal region and has five putative GTPase sites [2]. In our study, we identified four MEN1 germline variants
(c.564delC, c.1268G > A, IVS5 + 5delG, and c.1546_1547insC). Among them, c.564delC and c.1546_1547insC are frameshift variants, which are predicted to result in truncated forms of menin. This leads to partial or complete loss of the NLSs and results in loss of the ability to transfer through the nuclear envelope $[3,11]$. The c.1546_1547insC variant has been reported to be a potential mutational hotspot with a frequency of $2.7 \%$ in MEN1 families [12, 13]. The c. $1268 \mathrm{G}>\mathrm{A}$ variant was previously reported in an Australian patient, who manifested lung and thymic carcinoids, prolactinoma, non-functioning pituitary, insulinoma, and hyperparathyroidism [10]. Considering the young age of patient \#2, close follow-up and further screening for additional manifestations are recommended. In our study, the novel splice variant IVS5 + 5delG resulted in the skipping of neighboring exons and the introduction of premature translation stop codons. Interestingly, another MEN1 variant IVS5 + 5G > A in the same locus was described as likely pathogenic in the ClinVar database. In-silico analysis predicted it may damage or destroy the natural donor site and lead to abnormal

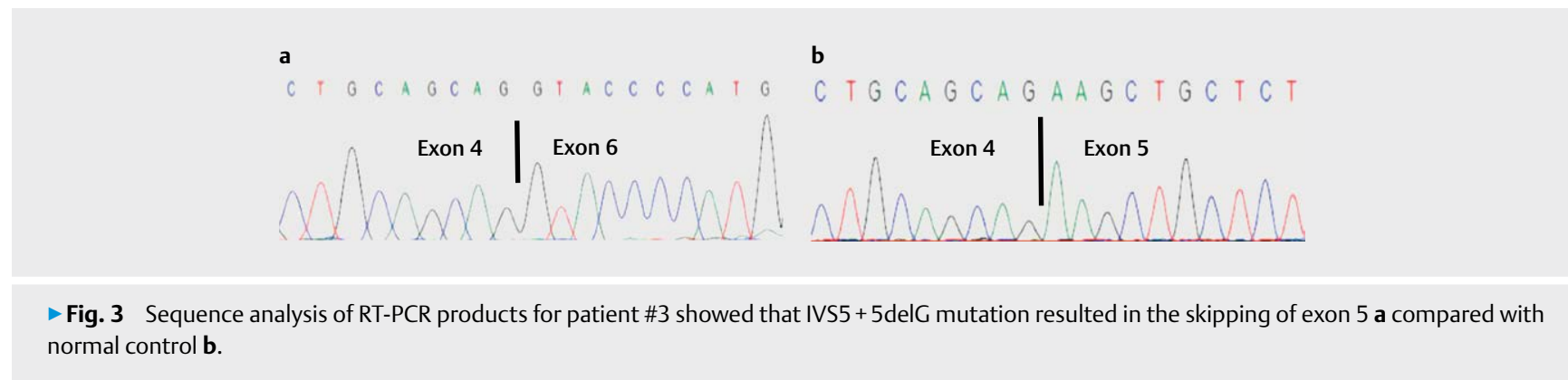

normal control $\mathbf{b}$.
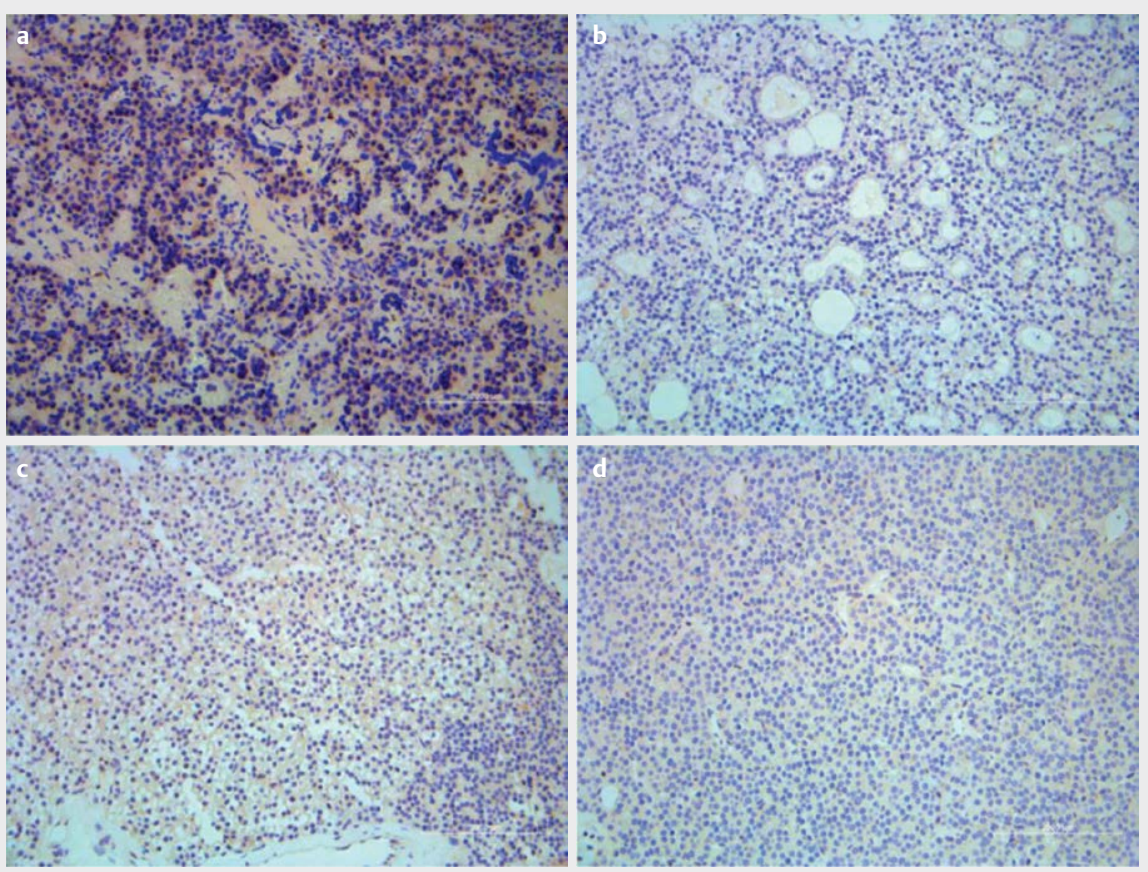

- Fig. 4 Immunohistochemistry for menin. Parathyroid hyperplasia in renal insufficiency without MEN1 mutation (a 20X). Parathyroid adenoma from patient \#1 (b 20X), patient \#2 (c 20X), and patient \#3 (d 20X). 
gene splicing, further suggesting that the novel IVS5 + 5delG splice variant lies in a biologically important region.

We identified another novel somatic variant, c.249_252delGTCT, and LOH of MEN1 locus C.564delC variants in the parathyroid tumoral tissues of patient \#1. Additionally, a novel somatic variant, c.313_314insC, and LOH of MEN1 IVS5 + 5delG were identified in the thymoma and parathyroid tumoral tissues of patient \#3, which might indicate the pathogenic role of these variants and support the Knudson's two-hit mechanism for MEN1 development. All the variants identified in our study are likely to result in a truncated protein. Therefore, we speculated that the menin protein was absent or the expression was reduced in the tumor. Indeed, reduced menin immunostaining was observed in the parathyroid adenoma tissues, which further supports our hypothesis. Owing to the small sample size of our study, the true pathogenicity of the variations still needs to be confirmed with a larger number of clinical cases. Further functional studies, such as animal models or cell work, are also needed to provide more evidence.

The variant p.Ala541Thr is usually considered a polymorphism, with an allelic frequency of $15 \%$ in the general population (Hap Map Database). The potential pathogenicity of the p.Ala541Thr variant is still controversial $[14,15]$. Nozières et al [15] compared a series of 55 patients carrying the p.Ala541Thr variant with a group of 117 MEN1 patients carrying other MEN1 variants and found the p.Ala541Thr variant may be involved in a low-penetrance MEN1 pheno-

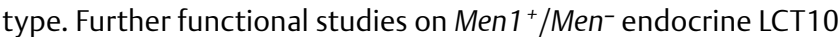
cells showed that the overexpression of the p.Ala541Thr variant did not inhibit cell growth, which is similar to the effect of mutant forms of the menin protein. However, there were some limitations in the Nozières study; for example, family information was limited in the p.Ala541Thr group, large MEN1 deletions and other gene (CDNK1B/p27) variants were not excluded, and their findings were mainly based on Caucasians [15]. In our study, patient \#3's father and sister both carried this variant but did not show any clinical manifestation of MEN1. Considering the high frequency of the p.Ala541Thr variant in Asiatic populations (up to $30 \%$ ) [15], additional studies are needed to investigate whether it may be pathogenic.

MEN1 penetrance is reported to be $50 \%$, above $95 \%$, and almost $100 \%$ at the age 20,40 , and after 70 , respectively [16]. The natural progression of MEN1 is long and hence, it is difficult to diagnose the disorder during its early stages. In our study, all four patients were diagnosed after the involvement of multiple glands, which leads to an unfavorable prognosis. Two of the patients received parathyroidectomy twice and one patient suffered from recurrent insulinoma even after undergoing surgery three times. Studies showed that MEN1-associated primary hyperparathyroidism usually involves two or more parathyroid glands, and occurs at a young age $[17,18]$. MEN1-associated insulinoma recurrence is four times more common than recurrence in individuals without MEN1 10 years after the first presentation [19]. Thymoma occurs only in 2.0$8.4 \%$ of MEN 1 patients. However, $22 \%$ and $89 \%$ of thymic carcinoid MEN1 patients experience synchronous and metachronous distant metastasis, and the 10-year overall survival rate is only $45 \%$ [20]. In our study, the pathology stage of thymoma was $\mathrm{G} 2$ in patient \#3 even though he was asymptomatic. Additionally, adjacent lymph node metastases were detected at the time of diagnosis, thus indicating a poor prognosis. Considering the high morbidity and mor- tality in MEN1, early clinical and genetic diagnosis of patients would help in effective disease management.

In conclusion, we have evaluated the clinical and genetic characteristics of four patients with MEN1 and identified four heterozygous MEN1 germline variants (c.564delC, c. 1268G > A, IVS5 + 5delG, and c.1546_1547insC) as well as two novel somatic variants (c.249_252delGTCT and c.313_314insC). Early recognition of the phenotype coupled with screening for the MEN1 gene is the key to early diagnosis and treatment of MEN1.

\section{Funding}

This work was supported by the National Natural Science Foundation of China (81670730, 81100583).

\section{Conflict of Interest}

The authors declare that they have no conflict of interest.

\section{References}

[1] Thakker RV, Newey PJ, Walls GV et al. Clinical Practice Guidelines for Multiple Endocrine Neoplasia Type 1 (MEN1). J Clin Endocrinol Metab 2012; 97: 2990-3011

[2] Lemos MC, Thakker RV. Multiple Endocrine Neoplasia Type 1 (MEN1): Analysis of 1336 mutations reported in the first decade following identification of the gene. Hum Mutat 2008; 29: 22-32

[3] Matkar S, Thiel A, Hua X. Menin: A scaffold protein that controls gene expression and cell signaling. Trends Biochem Sci 2013; 38: 394-402

[4] Kamilaris CDC, Stratakis CA. Multiple Endocrine Neoplasia Type 1 (MEN1): An update and the significance of early genetic and clinical diagnosis. Front Endocrinol (Lausanne) 2019; 10: 339

[5] Pannett AA, Thakker RV. Somatic mutations in MEN type 1 tumors, consistent with the Knudson "two-hit" hypothesis. J Clin Endocrinol Metab 2001; 86: 4371-4374

[6] Pellegata NS, Quintanilla-Martinez L, Siggelkow H et al. Germ-line mutations in p27Kip1 cause a multiple endocrine neoplasia syndrome in rats and humans. Proc Natl Acad Sci USA 2006; 17: 15558-15563

[7] Lee M, Pellegata NS. Multiple endocrine neoplasia syndromes associated with mutation of p27. J Endocrinol Invest 2013; 36: 781-787

[8] Alrezk R, Hannah-Shmouni F, Stratakis CA. MEN4 and mutations: The latest of the MEN syndromes. Endocr Relat Cancer 2017; 24: T195-T208

[9] Richards S, Aziz N, Bale S et al. Standards and guidelines for the interpretation of sequence variants: $A$ joint consensus recommendation of the American College of Medical Genetics and Genomics and the Association for Molecular Pathology. Genet Med 2015; 17: 405-424

[10] Bergman L, Teh B, Cardinal J et al. Identification of MEN1 gene mutations in families with MEN 1 and related disorders. Br J Cancer 2000; 83: 1009-1014

[11] Huang J, Gurung B, Wan B et al. The same pocket in menin binds both MLL and JUND but has opposite effects on transcription. Nature 2012; 12: 482 (7386): 542-546

[12] Thakker RV. Multiple endocrine neoplasia type 1 (MEN1). Best Pract Res Clin Endocrinol Metab 2010; 24: 355-370 
[13] Zha BB, Liang W, Liu J et al. Mutation analysis in a Chinese family with multiple endocrine neoplasia type 1. Chin Med J (Engl) 2010; 123: 569-573

[14] Bazzi W, Renon M, Vercherat C et al. MEN1 missense mutations impair sensitization to apoptosis induced by wild-type menin in endocrine pancreatic tumor cells. Gastroenterology 2008; 135: e2

[15] Nozières C, Zhang CX, Buffet A et al. p.Ala541Thr variant of MEN1 gene: A non deleterious polymorphism or a pathogenic mutation? Ann. Endocrinol (Paris) 2014; 75: 133-140

[16] Giacche M, Panarotto A, Mori L et al. A novel menin gene deletional mutation in a little series of Italian patients affected by apparently sporadic multiple endocrine neoplasia type 1 syndrome. J Endocrinol Invest 2012; 35: 124-128
[17] Lourenço DM Jr., Coutinho FL, Toledo RA et al. Early-onset, progressive, frequent, extensive, and severe bone mineral and renal complications in multiple endocrine neoplasia type 1-associated primary hyperparathyroidism. J Bone Miner Res 2010; 25: 2382-2391

[18] Twigt BA, Scholten A, Valk GD et al. Differences between sporadic and MEN related primary hyperparathyroidism; clinical expression, preoperative workup, operative strategy and follow-up. Orphanet J Rare Dis 2013; 8: 50

[19] Service F], McMahon MM, O'Brien PC et al. Functioning insulinomaincidence, recurrence, and long-term survival of patients: A 60-year study. Mayo Clin Proc 1991; 66: 711-719

[20] Christakis I, Qiu W, Silva Figueroa AM et al. Clinical features, treatments, and outcomes of patients with thymic carcinoids and multiple endocrine neoplasia type 1 syndrome at MD Anderson Cancer Center. Horm Cancer 2016; 7: 279-287 
Supplementary Material

- Table 1S PCR and sequencing primers for MEN1 gene and CDKN1B gene

\begin{tabular}{|c|c|c|c|c|}
\hline Gene & Exon & Primer & PCR product (bp) & $\mathrm{T}\left({ }^{\circ} \mathrm{C}\right)$ \\
\hline \multirow[t]{7}{*}{ MEN1 } & $\begin{array}{l}2 \mathrm{~F} \\
2 \mathrm{R}\end{array}$ & $\begin{array}{l}\text { ACCGACAAAAGAGGGGAAGC } \\
\text { GGAAAATAACACCTGCCGAACC }\end{array}$ & 742 & 68 \\
\hline & $\begin{array}{l}3 / 4 \mathrm{~F} \\
3 / 4 \mathrm{R}\end{array}$ & $\begin{array}{l}\text { TAAACAGGGAGAGAGTCTTTGAAC } \\
\text { TCAGAACCAACAGGGACCAC }\end{array}$ & 986 & 64 \\
\hline & $\begin{array}{l}5 / 6 F \\
5 / 6 R\end{array}$ & $\begin{array}{l}\text { GATAGGCTAAGGACCCGTTCTC } \\
\text { CAGGAAGGACAGTAAGCAGTGG }\end{array}$ & 531 & 68 \\
\hline & $\begin{array}{l}7 F \\
7 R\end{array}$ & $\begin{array}{l}\text { ATTGGGCTGGGCTTGAAAGTC } \\
\text { GAGGGTGGTTGGAAACTGATGG }\end{array}$ & 506 & 68 \\
\hline & $\begin{array}{l}8 \mathrm{~F} \\
8 \mathrm{R}\end{array}$ & 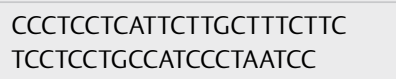 & 412 & 66 \\
\hline & $\begin{array}{l}9 F \\
9 R\end{array}$ & $\begin{array}{l}\text { GAATGTTCCCAACCTATGCTTACC } \\
\text { CCTCTCGGCTCACTATGCG }\end{array}$ & 545 & 60 \\
\hline & $\begin{array}{l}10 \mathrm{~F} \\
10 \mathrm{R}\end{array}$ & $\begin{array}{l}\text { ACTACAGGTGGTGACAGCAG } \\
\text { GAAAATCGTGGGTTTGATACAGAC }\end{array}$ & 834 & 68 \\
\hline \multirow[t]{4}{*}{ CDKN1B } & $1 \mathrm{~F}$ & TTGGCTCCGAGGGCAGTC & \multirow[t]{2}{*}{629} & \multirow[t]{2}{*}{64} \\
\hline & $1 R$ & ACACATTCTATGGTTGGGAAAGGG & & \\
\hline & $2 \mathrm{~F}$ & GGATTGTGGGTGGAGGTAGTG & \multirow[t]{2}{*}{308} & \multirow[t]{2}{*}{62} \\
\hline & $2 \mathrm{R}$ & GGTATCGTGAGGTCTGAAGGC & & \\
\hline
\end{tabular}

